\title{
Algebraic and Combinatorial Tools for State Complexity : Application to the Star-Xor Problem
}

\author{
Pascal Caron Edwin Hamel-de-le-court \\ Jean-Gabriel Luque \\ LITIS, Université de Rouen, \\ Avenue de l'Université, \\ 76801 Saint-Étienne du Rouvray Cedex, \\ France \\ \{Pascal.Caron, Edwin.Hamel-de-le-court, Jean-Gabriel.Luque\}@univ-rouen.fr
}

\begin{abstract}
We investigate the state complexity of the star of symmetrical differences using modifiers and monsters. A monster is an automaton in which every function from states to states is represented by at least one letter. A modifier is a set of functions allowing one to transform a set of automata into one automaton. These recent theoretical concepts allow one to find easily the desired state complexity. We then exhibit a witness with a constant size alphabet.
\end{abstract}

\section{Introduction}

The state complexity of a rational language is the size of its minimal automaton and the state complexity of a rational operation is the maximal one of those languages obtained by applying this operation onto languages of fixed state complexities.

The classical approach is to compute an upper bound and to provide a witness, that is a specific example reaching the bound which is then the desired state complexity.

Since the 70s, the state complexity of numerous unary and binary operations has been computed. See, for example, [9, 11, 14, 15, 16, 19] for a survey of the subject. More recently, the state complexity of combinations of operations has also been studied. In most cases the result is not simply the mathematical composition of the individual complexities and studies lead to interesting situations. Examples can be found in [7, 12, 17, 18].

In some cases, the classical method has to be enhanced by two independent approaches. The first one consists in describing states by combinatorial objects. Thus the upper bound is computed using combinatorial tools. For instance, in [4], the states are represented by tableaux representing boolean matrices and an upper bound for the catenation of symmetrical difference is given. These combinatorial objects will be used to compute an upper bound for the Kleene star of symmetrical difference. The second one is an algebraic method consisting in building a witness for a certain class of rational operations by searching in a set of automata with as many transition functions as possible. This method has the advantage of being applied to a large class of operations, but has the drawback of giving witnesses that have alphabets of nonconstant size. Witnesses with small alphabets are indeed favoured in this area of research when they can be found, as evidenced by several studies ([5, 6]). This approach has been described independently by Caron et al. in [3] as the monster approach and by Davies in [8] as the OLPA (One Letter Per Action) approach but was implicitly present in older papers like [2, 10].

J. Leroux and J.-F. Raskin (Eds.): Tenth International Symposium on Games, Automata, Logics, and Formal Verification (GandALF'19).

EPTCS 305, 2019, pp. 154-168, doi $10.4204 /$ EPTCS.305.11
(C) Caron, Hamel-de le Court \& Luque

This work is licensed under the Creative Commons Attribution License. 
In this paper, we illustrate these approaches to find the state complexity of the star of symmetrical difference. Furthermore, we improve the witness found by drastically reducing the size of its alphabet to a constant size.

The paper is organized as follows. Section 2 gives definitions and notations about automata and combinatorics. In Section 3, we recall the monster approach : we define modifiers, monsters, and give some properties of these structures related to state complexity. In Section 5 , the state complexity of star of symmetrical difference is computed. Hence, in Section 6, we find witnesses for this operation with an alphabet size of 17.

\section{Preliminaries}

\subsection{Operations over sets}

The cardinality of a finite set $E$ is denoted by \#E, the set of subsets of $E$ is denoted by $2^{E}$ and the set of mappings of $E$ into itself is denoted by $E^{E}$. The symmetric difference of two sets $E_{1}$ and $E_{2}$ is denoted by $\oplus$ and defined by $E_{1} \oplus E_{2}=\left(E_{1} \cup E_{2}\right) \backslash\left(E_{1} \cap E_{2}\right)$. For any positive integer $n$, let us denote $\{0, \ldots, n-1\}$ by $\llbracket n \rrbracket$. $\mathbb{1}$ denotes the identity mapping, the set of which depends on context.

\subsection{Languages and automata}

Let $\Sigma$ denote a finite alphabet. A word $w$ over $\Sigma$ is a finite sequence of symbols of $\Sigma$. The length of $w$, denoted by $|w|$, is the number of occurrences of symbols of $\Sigma$ in $w$. For $a \in \Sigma$, we denote by $|w|_{a}$ the number of occurrences of $a$ in $w$. The set of all finite words over $\Sigma$ is denoted by $\Sigma^{*}$. A language is a subset of $\Sigma^{*}$.

A complete and deterministic finite automaton (DFA) is a 5-tuple $A=(\Sigma, Q, i, F, \delta)$ where $\Sigma$ is the input alphabet, $Q$ is a finite set of states, $i \in Q$ is the initial state, $F \subseteq Q$ is the set of final states and $\delta$ is the transition function from $Q \times \Sigma$ to $Q$ extended in a natural way from $Q \times \Sigma^{*}$ to $Q$. The cardinality of $A$ is the cardinality of its set of states, i.e. $\# A=\# Q$. We will often use $\llbracket n \rrbracket$ for some $n \in \mathbb{N}$ as the set of states for DFAs.

Let $A=(\Sigma, Q, i, F, \delta)$ be a DFA. A word $w \in \Sigma^{*}$ is recognized by the DFA $A$ if $\delta(i, w) \in F$. The language recognized by a DFA $A$ is the set $\mathrm{L}(A)$ of words recognized by $A$. Two DFAs are said to be equivalent if they recognize the same language.

For any word $w$, we denote by $\delta^{w}$ the function $q \rightarrow \delta(q, w)$. Two states $q_{1}, q_{2}$ of $D$ are equivalent if for any word $w$ of $\Sigma^{*}, \delta\left(q_{1}, w\right) \in F$ if and only if $\delta\left(q_{2}, w\right) \in F$. This equivalence relation is called the Nerode equivalence and is denoted by $q_{1} \sim_{\mathrm{Ner}} q_{2}$. If two states are not equivalent, then they are called distinguishable.

A state $q$ is accessible in a DFA if there exists a word $w \in \Sigma^{*}$ such that $q=\delta(i, w)$. A DFA is minimal if there does not exist any equivalent DFA with less states and it is well known that for any DFA, there exists a unique minimal equivalent one ([13]). Such a minimal DFA can be obtained from $D$ by computing $\widehat{A}_{/ \sim}=\left(\Sigma, Q / \sim,[i], F / \sim, \delta_{\sim}\right)$ where $\widehat{A}$ is the accessible part of $A$, and where, for any $q \in Q,[q]$ is the $\sim$-class of the state $q$ and satisfies the property $\delta_{\sim}([q], a)=[\delta(q, a)]$, for any $a \in \Sigma$. The number of its states is denoted by $\#_{\text {Min }}(A)$. In a minimal DFA, any two distinct states are pairwise distinguishable.

Let $L$ be a regular language defined over an alphabet $\Sigma$. We denote by $L^{*}\left\{w=u_{1} \cdots u_{n} \mid u_{i} \in\right.$ $L \wedge n \in \mathbb{N}\}$. 
The syntactic semigroup of $L$ is the semigroup generated by the transition functions of all letters of the minimal DFA of $L$.

\subsection{State complexity}

A unary regular operation is a function from regular languages into regular languages of $\Sigma$. A $k$-ary regular operation over the alphabet $\Sigma$ is a function from the set of $k$-tuples of regular languages of $\Sigma$ into regular languages of $\Sigma$.

The state complexity of a regular language $L$ denoted by $\operatorname{sc}(L)$ is the number of states of its minimal DFA. This notion extends to regular operations: the state complexity of a unary regular operation $\otimes$ is the function $\mathrm{sc}_{\otimes}$ such that, for all $n \in \mathbb{N} \backslash\{\nvdash\}, \mathrm{sc}_{\otimes}(n)$ is the maximum of all the state complexities of $\otimes(L)$ when $L$ is of state complexity n, i.e. $\operatorname{sc}_{\otimes}(n)=\max \{\operatorname{sc}(\otimes(L)) \mid \operatorname{sc}(L)=n\}$.

This can be generalized, and the state complexity of a $k$-ary operation $\otimes$ is the $k$-ary function $\mathrm{sc} \otimes$ such that, for all $\left(n_{1}, \ldots, n_{k}\right) \in\left(\mathbb{N}^{*}\right)^{k}$,

$$
\mathrm{sc}_{\otimes}\left(n_{1}, \ldots, n_{k}\right)=\max \left\{\mathrm{sc}\left(\otimes\left(L_{1}, \ldots, L_{k}\right)\right) \mid \text { for all } i \in\{1, \ldots, k\}, \mathrm{sc}\left(L_{i}\right)=n_{i}\right\} .
$$

Then, a witness for $\otimes$ is a a way to assign to each $\left(n_{1}, \ldots, n_{k}\right)$, assumed sufficiently big, a k-tuple of languages $\left(L_{1}, \ldots, L_{k}\right)$ with $\operatorname{sc}\left(L_{i}\right)=n_{i}$, for all $i \in\{1, \ldots, k\}$, satisfying $\operatorname{sc}_{\otimes}\left(n_{1}, \ldots, n_{k}\right)=$ $\mathrm{sc}\left(\otimes\left(L_{1}, \ldots, L_{k}\right)\right)$.

\subsection{Morphisms}

Let $\Sigma$ and $\Gamma$ be two alphabets. A morphism is a function $\phi$ from $\Sigma^{*}$ to $\Gamma^{*}$ such that, for all $w, v \in \Sigma^{*}, \phi(w v)=\phi(w) \phi(v)$. Notice that $\phi$ is completely defined by its value on letters.

Let $L$ be a regular language over alphabet $\Sigma$ recognized by the DFA $A=(\Sigma, Q, i, F, \delta)$ and let $\phi$ be a morphism from $\Gamma^{*}$ to $\Sigma^{*}$. Then, $\phi^{-1}(L)$ is the regular language recognized by the DFA $B=\left(\Gamma, Q, i, F, \delta^{\prime}\right)$ where, for all $a \in \Gamma$ and $q \in Q, \delta^{\prime}(q, a)=\delta(q, \phi(a))$. Therefore, note that we have

Property 1 Let $L$ be a regular language and $\phi$ be a morphism. We have $\operatorname{sc}\left(\phi^{-1}(L)\right) \leq \operatorname{sc}(L)$.

We say that a morphism $\phi$ is 1-uniform if the image by $\phi$ of any letter is a letter. In other words, a 1-uniform morphism is a (not necessarily injective) renaming of the letters and the only complexity of the mapping stems from mapping $a$ and $b$ to the same image, i.e., $\phi(a)=\phi(b)$.

\section{Monsters and state complexity}

In [1], Brzozowski gives a series of properties that would make a language $L_{n}$ of state complexity $n$ sufficiently complex to be a good candidate for constructing witnesses for numerous classical rational operations. One of these properties is that the size of the syntactic semigroup is $n^{n}$, which means that each transformation of the minimal DFA of $L_{n}$ can be associated to a transformation by some non-empty word. This upper bound is reached when the set of transition functions of the DFA is exactly the set of transformations from state to state. We thus consider the set of transformations of $\llbracket n \rrbracket$ as an alphabet where each letter is simply named by the transition function it defines. This leads to the following definition :

Definition 1 A 1-monster is an automaton $\operatorname{Mon}_{n}^{F}=(\Sigma, \llbracket n \rrbracket, 0, F, \delta)$ defined by

- the alphabet $\Sigma=\llbracket n \rrbracket^{\llbracket n \rrbracket}$, 


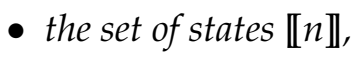

- the initial state 0 ,

- the set of final states $F$,

- the transition function $\delta$ defined for any $a \in \Sigma$ by $\delta(q, a)=a(q)$.

The language recognized by a 1-monster DFA is called a 1-monster language.

Example 1 The 1-monster $\operatorname{Mon}_{2}^{\{1\}}$ is

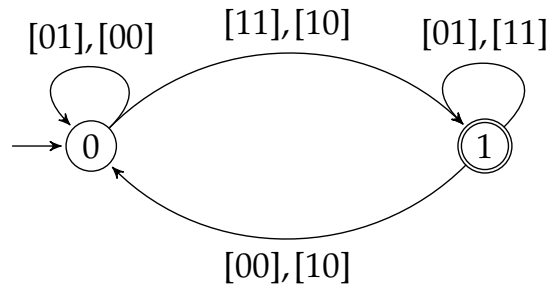

where, for all $i, j \in\{0,1\}$, the label $[i j]$ denotes the transformation sending 0 to $i$ and 1 to $j$, which is also a letter in the DFA above.

Let us notice that some families of 1-monster languages are witnesses for the Star and Reverse operations ([3]). The following claim is easy to prove and captures a universality-like property of 1-monster languages:

Property 2 Let $L$ be any regular language recognized by a DFA $A=(\Sigma, \llbracket n \rrbracket, 0, F, \delta)$. The language $L$ is the preimage of $\mathrm{L}\left(\mathrm{Mon}_{n}^{F}\right)$ by the 1-uniform morphism $\phi$ such that, for all $a \in \Sigma, \phi(a)=\delta^{a}$, i.e.

$$
L=\phi^{-1}\left(\operatorname{L}_{\left.\left(\operatorname{Mon}_{n}^{F}\right)\right)}\right.
$$

This is an important and handy property that we should keep in mind. We call it the restriction-renaming property.

We can wonder whether we can extend the notions above to provide witnesses for $k$-ary operators. In the unary case, the alphabet of a monster is the set of all possible transformations we can apply on the states. In the same mindset, a $k$-monster DFA is a $k$-tuple of DFAs, and its construction must involve the set of $k$-tuples of transformations as an alphabet. Indeed, the alphabet of a $k$-ary monster has to encode all the transformations acting on each set of states independently one from the others. This leads to the following definition :

Definition 2 A k-monster is a k-tuple of automata $\operatorname{Mon}_{n_{1}, \ldots, n_{k}}^{F_{1}, \ldots, F_{k}}=\left(\mathbb{M}_{1}, \ldots, \mathbb{M}_{k}\right)$ where $\mathrm{M}_{j}=\left(\Sigma, \llbracket n_{j} \rrbracket, 0, F_{j}, \delta_{j}\right)$ for $j \in\{1, k\}$ is defined by

- the common alphabet $\Sigma=\llbracket n_{1} \rrbracket^{\llbracket n_{1} \rrbracket} \times \ldots \times \llbracket n_{k} \rrbracket^{\llbracket n_{k} \rrbracket}$,

- the set of states $\llbracket n_{j} \rrbracket$,

- the initial state 0 ,

- the set of final states $F_{j}$,

- the transition function $\delta_{j}$ defined for any $\left(a_{1}, \ldots, a_{k}\right) \in \sum$ by $\delta_{j}\left(q,\left(a_{1}, \ldots, a_{k}\right)\right)=a_{j}(q)$.

A $k$-tuple of languages $\left(L_{1}, \ldots, L_{k}\right)$ is called a monster $k$-language if there exists a $k$-monster

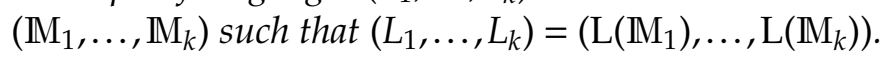


Remark 1 When $F_{j}$ is different from $\emptyset$ and $Q_{j}, \mathrm{M}_{j}$ is minimal.

Definition 2 allows us to extend the restriction-renaming property in a way that is still easy to check.

Property 3 Let $\left(L_{1}, \ldots, L_{k}\right)$ be a k-tuple of regular languages over the same alphabet $\Sigma$. We assume that each $L_{j}$ is recognized by the DFA $A_{j}=\left(\sum, \llbracket n_{j} \rrbracket, 0, F_{j}, \delta_{j}\right)$. Let $\operatorname{Mon}_{n_{1}, \ldots, n_{k}}^{F_{1}, \ldots, F_{k}}=\left(\mathbb{M}_{1}, \ldots, \mathbb{M}_{k}\right)$. For all $j \in\{1, \ldots, k\}$, the language $L_{j}$ is the preimage of $L\left(\mathbb{M}_{j}\right)$ by the 1-uniform morphism $\phi$ such that, for all $a \in \Sigma, \phi(a)=\left(\delta_{1}^{a}, \ldots, \delta_{k}^{a}\right)$, i.e.

$$
\left(L_{1}, \ldots, L_{k}\right)=\left(\phi^{-1}\left(\mathrm{~L}\left(\mathbb{M}_{1}\right)\right), \ldots, \phi^{-1}\left(\mathrm{~L}_{\left.\left(\mathrm{M}_{k}\right)\right)}\right)\right.
$$

It has been shown that some families of 2-monsters are witnesses for binary boolean operations and for the catenation operation [3]. Many papers concerning state complexity actually use monsters as witnesses without naming them (e.g. [2]). Therefore, a natural question arises : can we define a simple class of rational operations for which monsters are always witnesses? This class should ideally encompass some classical regular operations, in particular the operations studied in the papers cited above. In the next section, we define objects that allow us to answer this question.

\section{Modifiers}

We first describe a class of regular operations for which monsters are always witnesses in the unary case. Once again, the restriction-renaming property comes in handy and gives us the intuition we need. We call 1-uniform any unary regular operation $\otimes$ that commutes with any 1-uniform morphism, i.e. for every regular language $L$ and every 1-uniform morphism $\phi$, $\otimes\left(\phi^{-1}(L)\right)=\phi^{-1}(\otimes(L))$. For example, it is proven in [8] that the Kleene star and the reverse are 1-uniform. Suppose now that $\otimes$ is a unary 1-uniform operation. Then, if $L$ is a regular language, $A=(\Sigma, \llbracket n \rrbracket, 0, F, \delta)$ its minimal DFA, and $\phi$ the 1-uniform morphism sending any letter of $\Sigma$ into its associated transition function in $A$, we have

$$
\otimes(L)=\otimes\left(\phi^{-1}\left(\operatorname{L} \operatorname{Mon}_{n}^{F}\right)\right)=\phi^{-1}\left(\otimes \left(\operatorname{L}_{\left.\left.\left(\operatorname{Mon}_{n}^{F}\right)\right)\right)}\right.\right.
$$

It follows that $\mathrm{sc}(\otimes(L))=\mathrm{sc}\left(\phi^{-1}\left(\otimes\left(\operatorname{L}\left(\operatorname{Mon}_{n}^{F}\right)\right)\right)\right) \leq \mathrm{sc}\left(\otimes\left(\mathrm{L}_{\left(\mathrm{Mon}_{n}\right.}^{F}\right)\right)$ by Property 1. In addition, Remark 1 implies that $\mathrm{L}\left(\operatorname{Mon}_{n}^{F}\right)$ has the same state complexity as $L$. Therefore, we have

Theorem 1 Any 1-uniform operation admits a family of monster 1-languages as a witness.

We now introduce the second central concept of our paper. In many cases, to compute state complexities, it is easier to describe regular operations as constructions on DFAs. We would therefore like to find a class of operations on DFAs, that are naturally associated to 1-uniform operations. Such an operation on DFAs needs to have some constraints that are described in the following definitions.

Definition 3 The state configuration of a DFA $A=(\Sigma, Q, i, F, \delta)$ is the triplet $(Q, i, F)$.

Definition 4 A 1-modifier is a unary operation on DFA $\mathrm{m}$ that produces a DFA such that:

- For any DFA $A$, the alphabet of $\mathrm{m}(A)$ is the same as the alphabet of $A$. 
- For any DFA $A$, the state configuration of $\mathrm{m}(A)$ depends only on the state configuration of the DFA A.

- For any DFA $A$ over the alphabet $\Sigma$, for any letter $a \in \Sigma$, the transition function of $a$ in $\mathrm{m}(A)$ depends only on the state configuration of the DFA $A$ and on the transition function of a in $A$.

Example 2 The star modifier. For all DFA $A=(\Sigma, Q, i, F, \delta)$, define $\operatorname{Star}(A)=\left(\Sigma, 2^{Q}, \emptyset,\{E \mid E \cap F \neq\right.$ $\left.\emptyset\} \cup\{\emptyset\}, \delta_{1}\right)$, where $\delta_{1}$ is as follows : for all $a \in \Sigma$,

$$
\delta_{1}^{a}(\emptyset)=\left\{\begin{array}{l}
\left\{\delta^{a}(i)\right\} \text { if } \delta^{a}(i) \notin F \\
\left\{\delta^{a}(i), i\right\} \text { otherwise }
\end{array} \quad \text { and, for all } E \neq \emptyset, \delta_{1}^{a}(E)=\left\{\begin{array}{l}
\delta^{a}(E) \text { if } \delta^{a}(E) \cap F=\emptyset \\
\delta^{a}(E) \cup\{i\} \text { otherwise }
\end{array}\right.\right.
$$

The modifier Star describes the classical construction on DFA associated to the Star operation on languages, i.e. for all DFA $A, \mathrm{~L}(A)^{*}=\mathrm{L}($ Star $(A))$.

Example 3 If we apply the modifier Star to the modifier $\operatorname{Mon}_{2}^{\{1\}}$ described in Example 1 , we obtain the DFA drawn in Figure 1

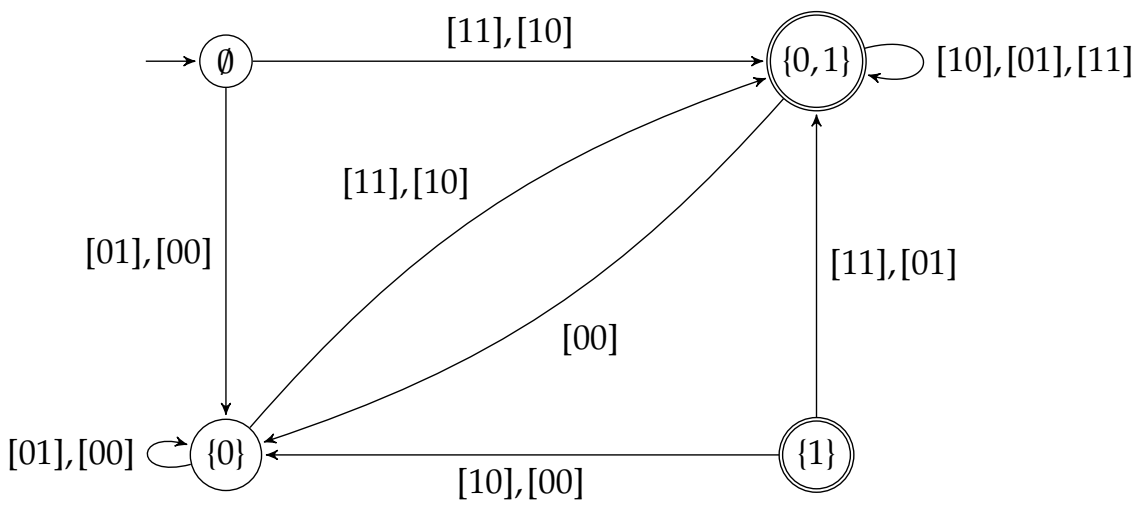

Figure 1: $\operatorname{star}\left(\operatorname{Mon}_{2}^{\{1\}}\right)$

From this, one deduces the action of the modifier Star on any DFA with two states. For instance, applying Star to DFA C (Figure 2) gives the DFA described in Figure 3

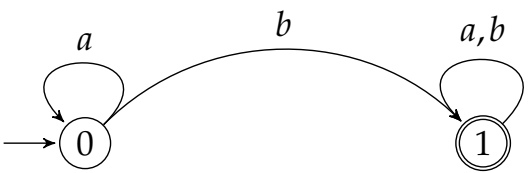

Figure 2: The DFA C

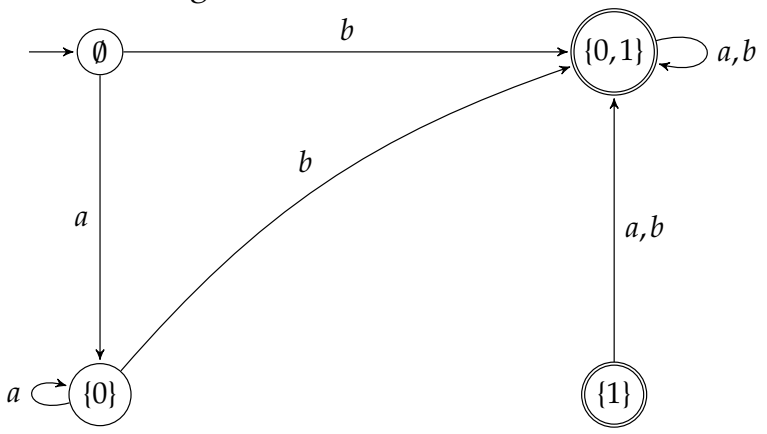

Figure 3: $\operatorname{star}(C)$

Remark that to apply Star to $C$, we just take the subautomaton of $\operatorname{Star}\left(\operatorname{Mon}_{2}^{\{1\}}\right)$ with letters being exactly the transition functions of letters in $C$, and rename its letters by the letters of $C$ of which they are the transition functions. The transition labeled by $b$ in Figure 2 is first assimilated to the transition [11] in $\operatorname{Mon}_{2}^{\{1\}}$ (see Example 1). Hence, the transition labeled by $b$ in $\operatorname{Gtar}(C)$ is the same as the transition labeled by [11] in Star(Mon ${ }_{2}^{\{1\}}$ ) (Figure 1). 
Theorem 2 A regular unary operation $\otimes$ is 1-uniform if and only if there exists a 1-modifier $m$ such that for any regular language $L$ and any DFA A recognizing $L, \otimes(L)=\mathrm{L}(\mathrm{m}(A))$.

Proof: Let $\otimes$ be a 1-uniform unary operation. We define a 1-modifier $m$ as follows. For any DFA $A=\left(\Sigma, Q_{A}, i_{A}, F_{A}, \delta_{A}\right)$, we can rename its set of states so that $A$ becomes the DFA $D=(\Sigma, \llbracket n \rrbracket, 0, F, \delta)$. Let us denote by $B=\left(\llbracket n \rrbracket^{\llbracket n \rrbracket}, Q^{\prime}, i^{\prime}, F^{\prime}, \delta^{\prime}\right)$ the minimal DFA of $\otimes\left(\mathrm{L}\left(\mathrm{Mon}_{n}^{F}\right)\right)$. We set $\mathfrak{m}(A)=\left(\Sigma, Q^{\prime}, i^{\prime}, F^{\prime}, \tilde{\delta}^{\prime}\right)$, with $\tilde{\delta}^{\prime}(q, a)=\delta^{\prime}\left(q, \delta^{a}\right)$. Notice that $\mathfrak{m}$ is indeed a 1-modifier. First, $\left(Q^{\prime}, i^{\prime}, F^{\prime}\right)$ depends only on $\left(Q_{A}, i_{A}, F_{A}\right)$. Second, $\tilde{\delta}^{\prime a}$ depends only on $\delta^{a}$ and on $\delta^{\prime}$, which in turn depend only on $\left(Q_{A}, i_{A}, F_{A}\right)$ and $\delta_{A}^{a}$.

Furthermore, by construction, $\mathrm{L}(\mathrm{m}(A))=\phi^{-1}(\mathrm{~L}(B))$, where $\phi$ is the 1-uniform morphism such that $\phi(a)=\delta_{D}^{a}$ for all $a \in \Sigma$. Therefore, we have $\mathrm{L}(\mathrm{m}(A))=\phi^{-1}\left(\otimes\left(\mathrm{L}\left(\operatorname{Mon}_{n}^{F}\right)\right)\right)$. And, since $\otimes$ is 1-uniform, we obtain $\mathrm{L}(\mathfrak{m}(A))=\otimes\left(\phi^{-1}\left(\mathrm{~L}\left(\operatorname{Mon}_{n}^{F}\right)\right)\right)=\otimes(L)$.

Conversely, let $\otimes$ be a regular operation and let $m$ be a 1-modifier such that for any regular language $L$ and any DFA $A$ recognizing $L, \otimes(L)=\mathrm{L}(\mathfrak{m}(A))$. We must prove that $\otimes$ is 1-uniform. Let $\Gamma$ and $\Sigma$ be two alphabets. Consider a 1-uniform morphism $\phi$ from $\Gamma^{*}$ to $\Sigma^{*}$ and a language $L$ over $\Sigma$. Let $A=(\Sigma, Q, i, F, \delta)$ be any DFA recognizing $L$ and let $B=(\Gamma, Q, i, F, \tilde{\delta})$ the DFA such that $\tilde{\delta}^{a}=\delta^{\phi(a)}$ for any letter $a \in \Gamma$. We have $\mathrm{L}(B)=\phi^{-1}(\mathrm{~L}(A))$.

Let $\mathfrak{m}(A)=\left(\Sigma, Q_{1}, i_{1}, F_{1}, \delta_{1}\right)$ and $\mathfrak{m}(B)=\left(\Gamma, Q_{2}, i_{2}, F_{2}, \delta_{2}\right)$. Since the state configuration of $A$ is the same as the state configuration of $B$, we have $\left(Q_{1}, i_{1}, F_{1}\right)=\left(Q_{2}, i_{2}, F_{2}\right)$. Furthermore, because the transition function of any letter $a \in \Gamma$ in $B$ is also the same as the transition function of $\phi(a)$ in $A$, we have $\delta_{2}^{a}=\delta_{1}^{\phi(a)}$. Hence, $\mathrm{L}(\mathfrak{m}(B))=\phi^{-1}(\mathrm{~L}(\mathfrak{m}(A)))$, which implies that $\otimes(\mathrm{L}(B))=\phi^{-1}(\otimes(A))$. Therefore, $\otimes\left(\phi^{-1}(\mathrm{~L}(A))\right)=\phi^{-1}(\otimes(\mathrm{L}(A)))$, as expected.

We extend the previous theorems by generalizing the definitions to $k$-ary operations.

Definition 5 A $k$-ary regular operation $\otimes$ is called 1-uniform if, for any $k$-tuple of rational languages $\left(L_{1}, \ldots, L_{k}\right)$, for any 1-uniform morphism $\phi, \otimes\left(\phi^{-1}\left(L_{1}\right), \ldots, \phi^{-1}\left(L_{k}\right)\right)=\phi^{-1}\left(\otimes\left(L_{1}, \ldots, L_{k}\right)\right)$.

Using the same arguments as in Theorem 1, we find

Theorem 3 Any k-ary 1-uniform operation admits a family of monster k-languages as a witness.

Proof: Suppose now that $\otimes$ is a $k$-ary 1-uniform operation. Then, if $\left(L_{1}, \ldots, L_{k}\right)$ is a $k$-tuple of regular languages over $\Sigma,\left(A_{1}, \ldots, A_{k}\right)$ the $k$-tuple of DFAs such that each $A_{j}=\left(\Sigma, Q_{j}, i_{j}, F_{j}, \delta_{j}\right)$ is the minimal DFA of $L_{i}$, and $\phi$ the 1-uniform morphism such that, for all $a \in \Sigma, \phi(a)=\left(\delta_{1}^{a}, \ldots, \delta_{k}^{a}\right)$, and if $\operatorname{Mon}_{n_{1}, \ldots, n_{k}}^{F_{1}, \ldots, F_{k}}=\left(\mathbb{M}_{1}, \ldots, \mathbb{M}_{k}\right)$, then $\left.\otimes(L)=\otimes\left(\phi^{-1}\left(\mathrm{~L}_{\left(\mathrm{M}_{1}\right)}\right), \ldots, \phi^{-1}\left(\mathrm{~L}\left(\mathrm{M}_{k}\right)\right)\right)=\phi^{-1}\left(\otimes\left(\mathrm{L}_{\left(\mathrm{M}_{1}\right)}\right) \ldots, \mathrm{L}\left(\mathbb{M}_{k}\right)\right)\right)$. It follows that $\left.\mathrm{sc}(\otimes(L))=\mathrm{sc}\left(\phi^{-1}\left(\otimes\left(\mathrm{L}\left(\mathbb{M}_{1}\right), \ldots, \mathrm{L}\left(\mathbb{M}_{k}\right)\right)\right)\right) \leq \mathrm{sc}\left(\otimes\left(\mathrm{L}_{\left(\mathrm{M}_{1}\right)}\right) \ldots, \mathrm{L}\left(\mathbb{M}_{k}\right)\right)\right)$ by Property 1 In addition, each $\mathrm{L}\left(\mathrm{M}_{j}\right)$ has the same state complexity as $L_{j}$.

Definition 6 A k-modifier is a k-ary operation on DFAs over the same alphabet that returns a DFA and such that :

- The alphabet of $\mathrm{m}\left(A_{1}, \ldots, A_{k}\right)$ is the same as the alphabet of each $A_{j}$.

- For any $k$-tuple of DFAs $\left(A_{1}, \ldots, A_{k}\right)$, the state configuration of $\mathrm{m}\left(A_{1}, \ldots, A_{k}\right)$ depends only on the state configurations of the DFAs $A_{1}, \ldots, A_{k}$.

- For any $k$-tuple of DFAs $\left(A_{1}, \ldots, A_{k}\right)$ where each DFA is over the alphabet $\Sigma$, for any letter $a \in \Sigma$, the transition function of a in $\mathrm{m}\left(A_{1}, \ldots, A_{k}\right)$ depends only on the state configurations of the DFAs $A_{1}, \ldots, A_{k}$ and on the transition functions of $a$ in each of the DFAs $A_{1}, \ldots, A_{k}$. 
Example 4 For all DFAs $A=\left(\Sigma, Q_{1}, i_{1}, F_{1}, \delta_{1}\right)$ and $B=\left(\Sigma, Q_{2}, i_{2}, F_{2}, \delta_{2}\right)$, define

$$
\mathfrak{X} \mathfrak{p r}(A, B)=\left(\Sigma, Q_{1} \times Q_{2},\left(i_{1}, i_{2}\right),\left(F_{1} \times\left(Q_{2} \backslash F_{2}\right) \cup\left(Q_{1} \backslash F_{1}\right) \times F_{2}\right),\left(\delta_{1}, \delta_{2}\right)\right)
$$

The modifier $\mathfrak{X} \mathfrak{o r}$ describes the classical construction associated to the operation Xor on couples of languages, i.e for all DFAs $A$ and $B, \mathrm{~L}(A) \oplus \mathrm{L}(B)=\mathrm{L}(\mathfrak{X} \mathfrak{o r}(A, B))$.

Theorem 4 A regular $k$-ary operation $\otimes$ is 1-uniform if and only if there exists a $k$-modifier $m$ such that for any $k$-tuple of regular languages $\left(L_{1}, \ldots, L_{k}\right)$ and any $k$-tuple of DFAs $\left(A_{1}, \ldots, A_{k}\right)$ such that each $A_{j}$ recognizes $L_{j}$, we have $\otimes\left(L_{1}, \ldots, L_{k}\right)=\mathrm{L}\left(\mathfrak{m}\left(A_{1}, \ldots, A_{k}\right)\right)$.

The proof of Theorem 2 can be easily adapted to $k$-ary operations.

The following proposition states the effects of composition on modifiers and 1-uniform operations and directly stems from Definitions 5 and 6 .

Proposition 1 Let $\otimes_{1}$ be a $k_{1}$-ary 1-uniform operation and $\otimes_{2}$ be a $k_{2}$-ary 1-uniform operation. The $\left(k_{1}+k_{2}\right)$-ary operation defined by $\otimes\left(L_{1}, \ldots, L_{k_{1}+k_{2}}\right)=\otimes_{1}\left(L_{1}, \ldots, L_{l}, \otimes_{2}\left(L_{l+1}, \ldots, L_{l+k_{2}}\right), L_{l+k_{2}}, \ldots, L_{k_{1}+k_{2}}\right)$ is 1-uniform. Furthermore, if $\mathrm{m}_{1}$ is a $k_{1}$-modifier associated with $\otimes_{1}$ and $\mathrm{m}_{2}$ is a $k_{2}$-modifier associated with $\otimes_{2}$, the operation on $\left(k_{1}+k_{2}\right)$-tuples of DFAs defined by

$$
\mathfrak{m}\left(A_{1}, \ldots, A_{k_{1}+k_{2}}\right)=\mathfrak{m}_{1}\left(A_{1}, \ldots, A_{l}, \mathfrak{m}_{2}\left(A_{l+1}, \ldots, A_{l+k_{2}}\right), A_{l+k_{2}}, \ldots, A_{k_{1}+k_{2}}\right)
$$

is a modifier associated to $\otimes$.

\section{State complexity of the star of symmetrical difference}

In this section, we compute the state complexity of the 2-ary regular operation $L_{1} \circledast L_{2}=\left(L_{1} \oplus\right.$ $\left.L_{2}\right)^{*}$. Examples 2 and 4 together with Proposition 1 show that $\circledast$ is 1 -uniform and that an associated modifier can be defined by $\operatorname{St\mathfrak {X}}\left(A_{1}, A_{2}\right)=\operatorname{Star}\left(\mathfrak{X} \mathfrak{n r}\left(A_{1}, A_{2}\right)\right)$. To be more precise, if $A_{1}=\left(\Sigma, Q_{1}, i_{1}, F_{1}, \delta_{1}\right)$ and $A_{2}=\left(\Sigma, Q_{2}, i_{2}, F_{2}, \delta_{2}\right)$, then

$$
\operatorname{Gt} \mathfrak{X}\left(A_{1}, A_{2}\right)=\left(\sum, 2^{Q_{1} \times Q_{2}}, \emptyset,\left\{E \in 2^{Q_{1} \times Q_{2}} \mid E \cap F \neq \emptyset\right\} \cup\{\emptyset\}, \delta\right)
$$

where $F=\left(F_{1} \times Q_{2}\right) \oplus\left(Q_{1} \times F_{2}\right)$ and, for all $a \in \Sigma$,

$$
\delta^{a}(\emptyset)=\left\{\begin{array}{l}
\left\{\left(\delta_{1}^{a}\left(i_{1}\right), \delta_{2}^{a}\left(i_{2}\right)\right)\right\} \text { if }\left(\delta_{1}^{a}\left(i_{1}\right), \delta_{2}^{a}\left(i_{2}\right)\right) \notin F \\
\left\{\left(\delta_{1}^{a}\left(i_{1}\right), \delta_{2}^{a}\left(i_{2}\right)\right),\left(i_{1}, i_{2}\right)\right\} \text { otherwise }
\end{array}\right.
$$

and, for all $E \neq \emptyset, \delta^{a}(E)=\left\{\begin{array}{l}\left(\delta_{1}^{a}, \delta_{2}^{a}\right)(E) \text { if }\left(\delta_{1}^{a}, \delta_{2}^{a}\right)(E) \cap F=\emptyset \\ \left(\delta_{1}^{a}, \delta_{2}^{a}\right)(E) \cup\left\{\left(i_{1}, i_{2}\right)\right\} \text { otherwise. }\end{array}\right.$

Theorem 3 states that $\circledast$ admits a family of 2 -monsters as witness. For any positive integers

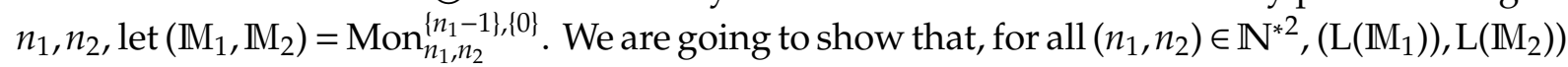
is indeed a witness for $\circledast$. This allows us to compute its state complexity. To be more precise, here is the outline of our proof. For any positive integers $n_{1}, n_{2}$, any $F_{1}, F_{2} \subseteq \llbracket n_{1} \rrbracket \times \llbracket n_{2} \rrbracket$, let us denote by $\mathrm{M}_{F_{1}, F_{2}}$ the DFA $\mathcal{S} \mathfrak{X}\left(\operatorname{Mon}_{n_{1}, n_{2}}^{F_{1}, F_{2}}\right)$. We are going to minimize the DFA $\mathrm{M}_{\left\{n_{1}-1\right\},\{0\}}$ by first computing its accessible states, and then, restricting it to its accessible states, by computing its Nerode equivalence. We will therefore have computed the minimal DFA equivalent to $\mathrm{M}_{\left\{n_{1}-1\right\},\{0\}}$, and computing its size allows us to compute the state complexity of $\mathrm{L}\left(\mathrm{M}_{\left\{n_{1}-1\right\},\{0\}}\right)$. We then show that the state complexity of $\mathrm{L}\left(\mathrm{M}_{\left\{n_{1}-1\right\},\{0\}}\right)$ is the greatest out of all the state complexities of $\mathrm{L}\left(\mathrm{M}_{F_{1}, F_{2}}\right)$, with $\left(F_{1}, F_{2}\right) \subseteq \llbracket n_{1} \rrbracket \times \llbracket n_{2} \rrbracket$. Theorem 3 allows us to conclude that the state complexity of $\mathrm{L}\left(\mathrm{M}_{\left\{n_{1}-1\right\},\{0\}}\right)$ is indeed $s c \circledast\left(n_{1}, n_{2}\right)$. 


\subsection{Computing the accessible states of $\mathrm{M}_{\left\{n_{1}-1\right\},\{0\}}$}

In order to understand more easily the next proofs, we associate elements of $2^{\llbracket n_{1} \rrbracket \times \llbracket n_{2} \rrbracket}$ to boolean matrices of size $n_{1} \times n_{2}$. Such a matrice is called a tableau when crosses are put in place of $1 \mathrm{~s}$, and 0 s are erased. We denote by the same letter the element of $2^{\llbracket n_{1} \rrbracket \times \llbracket n_{2} \rrbracket}$, the associated boolean matrix, and the associated tableau. If $T$ is an element of $2^{\llbracket n_{1} \rrbracket \times \llbracket n_{2} \rrbracket}$, we denote by $T_{x, y}$ the value of the boolean matrix $T$ at row $x$ and column $y$. Therefore, the three following assertions mean the same thing: a cross is at the coordinates $(x, y)$ in $T, T_{x, y}=1,(x, y) \in T$.

We say that a cross at coordinates $(x, y)$ in an element of $2^{\llbracket n_{1} \rrbracket \times \llbracket n_{2} \rrbracket}$ is in the final zone of $\mathrm{M}_{\mathrm{F}_{1}, \mathrm{~F}_{2}}$ if $(x, y) \in\left(F_{1} \times \llbracket n_{2} \rrbracket\right) \oplus\left(\llbracket n_{1} \rrbracket \times F_{2}\right)$. We remark that an element of $2^{\llbracket n_{1} \rrbracket \times \llbracket n_{2} \rrbracket}$ is final in $\mathrm{M}_{\mathrm{F}_{1}, \mathrm{~F}_{2}}$ if and only if it has a cross in the final zone of $\mathrm{M}_{\mathrm{F}_{1}, \mathrm{~F}_{2}}$. We fix for the remainder of this section two positive integers $n_{1}$ and $n_{2}$.

Lemma 1 The states of $\mathrm{M}_{\left\{n_{1}-1\right\},\{0\}}$ that are accessible are exactly the tableaux $T$ of size $n_{1} \times n_{2}$ such that, if $T$ has a cross in the final zone of $\mathrm{M}_{\left\{n_{1}-1\right\},\{0\}}$, then $T$ has cross at $(0,0)$.

Proof: It is easy to see by the definition of the transition function of St: that every tableau $T$ with a cross in the final zone of $\mathrm{M}_{\left\{n_{1}-1\right\},\{0\}}$ and no cross at $(0,0)$ is not accessible.

Let $\delta$ be the transition function of $\mathrm{M}_{\left\{n_{1}-1\right\},\{0\}}$. If $T$ is a tableau of size $n_{1} \times n_{2}$, let $\#_{\mathrm{nf}} T$ be the number of crosses of $T$ which are not in the final zone of $\mathrm{M}_{\left\{n_{1}-1\right\},\{0\}}$. Let us define an order $<$ on cross matrices as $T<T^{\prime}$ if and only if $\# T<\# T^{\prime}$ or $\left(\# T=\# T^{\prime}\right.$ and $\left.\#_{\mathrm{nf}} T<\#_{\mathrm{nf}} T^{\prime}\right)$.

Let us prove every tableau $T$ of size $n_{1} \times n_{2}$ such that, if $T$ has a cross in the final $\left(\left\{n_{1}-1\right\},\{0\}\right)$ zone, then $T$ has cross at $(0,0)$, is accessible by induction on non-empty cross matrices for the partial order < (the empty cross matrix is the initial state of $\mathrm{M}_{\left\{n_{1}-1\right\},\{0\}}$, and so it is accessible).

The only minimal cross matrix for non-empty matrices and the order $<$ is the cross matrix with only one cross at $(0,0)$. This is accessible from the initial state $\emptyset$ by reading the letter $(\mathbb{1}, \mathbb{1})$. Let us notice that each letter is a couple of functions of $\llbracket n_{1} \rrbracket^{\llbracket n_{1} \rrbracket} \times \llbracket n_{2} \rrbracket^{\llbracket n_{2} \rrbracket}$. Now let us take a cross matrix $T^{\prime}$, and find a cross matrix $T$ such that $T<T^{\prime}$, and $T^{\prime}$ is accessible from $T$. We distinguish the cases :

- $T^{\prime}$ has no cross in the final zone, except maybe at $(0,0)$.

- Case $T_{n_{1}-1,0}^{\prime}=0$. Let $(i, j)$ be the index of a cross of $T^{\prime}$. Let $(f, g)=((0, i),(0, j))$ where $(0, i)$ and $(0, j)$ denote transpositions, and let $T=(f, g)\left(T^{\prime}\right)$ where $(f, g)\left(T^{\prime}\right)=\{(f(i), g(j)) \mid$ $\left.(i, j) \in T^{\prime}\right\}$. As $(f, g)$ is a one-to-one transformation on $\llbracket n_{1} \rrbracket \times \llbracket n_{2} \rrbracket$, as $(f, g)\left(T^{\prime}\right)$ has a cross at $(0,0)$ and as $T^{\prime}$ does not have any crosses in the final zone, we have

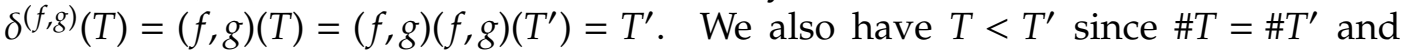
$\#_{\mathrm{nf}} T<\#_{\mathrm{nf}} T^{\prime}$.

- Case $T_{n_{1}-1,0}^{\prime}=1$. Let $(f, g)=\left(\left(0, n_{1}-1\right), \mathbb{1}\right)$ and let $T=(f, g)\left(T^{\prime}\right)$. We have $\delta^{(f, g)}(T)=T^{\prime}$, and $T<T^{\prime}$ as $\#_{\mathrm{nf}} T<\#_{\mathrm{nf}} T^{\prime}$.

- $T^{\prime}$ has a cross in the final zone other than $(0,0)$.

Let $(i, j)$ be such a cross, and let $(f, g)=((0, i),(0, j))$. Let $T^{\prime \prime}$ be the cross matrix obtained from $T^{\prime}$ by deleting the cross at $(0,0)$. Let $T=(f, g)\left(T^{\prime \prime}\right)$. As $(f, g)$ is still one-to-one on $\llbracket n_{1} \rrbracket \times \llbracket n_{2} \rrbracket$, we have $T_{0,0}=\left((f, g)\left(T^{\prime \prime}\right)\right)_{0,0}=T_{i, j}^{\prime \prime}=1$, and $((f, g)(T))=\left((f, g)\left((f, g)\left(T^{\prime \prime}\right)\right)\right)=T^{\prime \prime}$. As $T^{\prime \prime}$ has a cross in the final zone, we therefore have $\delta^{(f, g)}(T)=T^{\prime}$ and $T<T^{\prime}$ as \#T<\#T'.

- The only cross of $T^{\prime}$ which is in the final zone is $(0,0)$. 


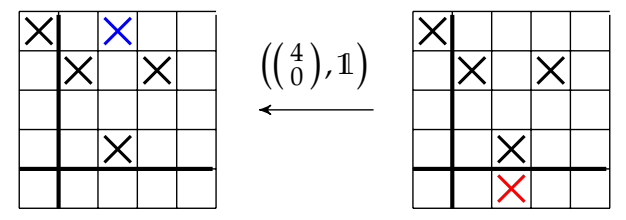

Figure 4: The two tableaux $T^{\prime}$ and $T$ for case $A$.

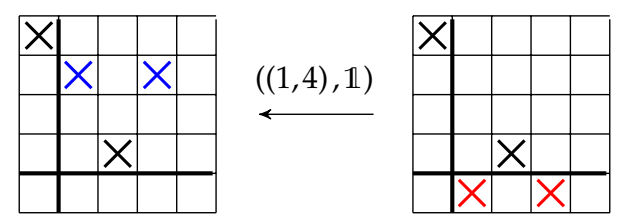

Figure 5: The two tableaux $T^{\prime}$ and $T$ for case $\neg A$ and $T_{n_{1}-1,0}^{\prime}=0$.

- Case A: there exists $j$ such that $T_{0, j}^{\prime}=1$.

Let $(f, g)=\left(\left(\begin{array}{cl}n_{1}-1 \\ 0\end{array}\right), \mathbb{1}\right)$ and let $T_{i, j}= \begin{cases}1 & \text { if }(i, j)=(0,0), \\ T_{0, j}^{\prime} & \text { if } i=n_{1}-1 \wedge j \neq 0, \\ T_{i, j}^{\prime} & \text { otherwise. }\end{cases}$

It is easy to check that $\delta^{(f, g)}(T)=T^{\prime}$, and $T<T^{\prime}$ as $\#_{\mathrm{nf}} T<\#_{\mathrm{nf}} T^{\prime}$.

- Case $\neg A$ and $T_{n_{1}-1,0}^{\prime}=0$. There exists $(i, j) \neq\left(n_{1}-1,0\right)$ such that $i \neq 0$ and $T_{i, j}^{\prime}=1$. Let $(f, g)=\left(\left(i, n_{1}-1\right), \mathbb{1}\right)$ and let $T=(f, g)\left(T^{\prime}\right)$. We have $\delta^{(f, g)}(T)=T^{\prime}$, and $T<T^{\prime}$ as $\#_{\mathrm{nf}} T<\#_{\mathrm{nf}} T^{\prime}$.

- Case $\neg A$ and $T_{n_{1}-1,0}^{\prime}=1$. Let $(f, g)=\left(\left(n_{1}-2, n_{1}-1\right), \mathbb{1}\right)$ and let $T=(f, g)\left(T^{\prime}\right)$. We have $\delta^{(f, g)}(T)=T^{\prime}$, and $T<T^{\prime}$ as $\#_{\mathrm{nf}} T<\#_{\mathrm{nf}} T^{\prime}$.

For all $\left(F_{1}, F_{2}\right) \subseteq \llbracket n_{1} \rrbracket \times \llbracket n_{2} \rrbracket$, let us now call $\widehat{\mathrm{M}}_{F_{1}, F_{2}}$ the DFA $\mathrm{M}_{F_{1}, F_{2}}$ restricted to states $T$ such that, if $T$ has a cross in the final zone of $\mathrm{M}_{F_{1}, F_{2}}$, then $T$ has cross at $(0,0)$. The following remark stems from the formula given for St:

Remark 2 The accessible part of $\mathrm{M}_{F_{1}, F_{2}}$ is included in $\widehat{\mathrm{M}}_{F_{1}, F_{2}}$.

\subsection{Computing the Nerode equivalence of $\widehat{\mathrm{M}}_{\left\{n_{1}-1\right\},\{0\}}$}

Definition $7 A$ tableau $T$ in $2^{\llbracket n_{1} \rrbracket \times \llbracket n_{2} \rrbracket}$ is right-triangle free if $\forall x, x^{\prime} \in \llbracket n_{1} \rrbracket$ such that $x \neq x^{\prime}$ and $\forall y, y^{\prime} \in \llbracket n_{2} \rrbracket$, such that $y \neq y^{\prime}$, we have $\#\left(\left\{(x, y),\left(x, y^{\prime}\right),\left(x^{\prime}, y\right),\left(x^{\prime}, y^{\prime}\right)\right\} \cap T\right) \neq 3$.

Definition 8 If $T$ and $T^{\prime}$ are distinct tableaux, we define the transformation on tableaux $\rightarrow$ as $T \rightarrow T^{\prime}$ if $T^{\prime}=T \cup\left\{\left(i^{\prime}, j^{\prime}\right)\right\}$, and there exists $(i, j)$ such that $\left\{(i, j),\left(i^{\prime}, j\right),\left(i, j^{\prime}\right)\right\} \subseteq T$. The equivalence relation $\stackrel{*}{\leftrightarrow}$ is defined as the symmetric, reflexive and transitive closure of $\rightarrow$.

For any tableau $T$, we define Sat $(T)$ as the smallest tableau (relatively to inclusion) with no right-triangle containing $T$. The existence and the unicity of Sat $(T)$ are easy to check. It is the representative of the equivalence class of $T$. Two tableaux $T$ and $T^{\prime}$ are therefore equivalent if $\operatorname{Sat}(T)=\operatorname{Sat}\left(T^{\prime}\right)$. 


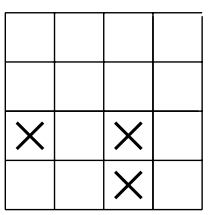

Figure 6: A tableau with a right-triangle

Lemma 2 The tableau $T$ in $2^{\llbracket n_{1} \rrbracket \times \llbracket n_{2} \rrbracket}$ is right-triangle free if and only if for all $i, i^{\prime} \in \llbracket n_{1} \rrbracket$, the lines $i$ and $i^{\prime}$ are either the same (for all $j \in \llbracket n_{2} \rrbracket, T_{i, j}=T_{i^{\prime}, j}$ ), or disjoint (for all $j \in \llbracket n_{2} \rrbracket, T_{i, j}=0 \vee T_{i^{\prime}, j}=0$ ).

Lemma 3 Let $\left(F_{1}, F_{2}\right) \subseteq \llbracket n_{1} \rrbracket \times \llbracket n_{2} \rrbracket$, and let $T$ and $T^{\prime}$ be any two states of $\mathrm{M}_{\mathrm{F}_{1}, \mathrm{~F}_{2}}$ such that $T \rightarrow T^{\prime}$. Then $T$ is final if and only if $T^{\prime}$ is final.

Let us recall that the alphabet of $\mathrm{M}_{\mathrm{F}_{1}, \mathrm{~F}_{2}}$ is $\llbracket n_{1} \rrbracket^{\llbracket n_{1} \rrbracket} \times \llbracket n_{2} \rrbracket^{\llbracket n_{2} \rrbracket}$. If $(f, g)$ is such a letter and $T=\left\{\left(x_{1}, y_{1}\right), \ldots,\left(x_{n}, y_{n}\right)\right\}$ is a tableau, then define $(f, g)(T)$ as $\left\{\left(f\left(x_{1}\right), g\left(y_{1}\right)\right), \ldots,\left(f\left(x_{n}\right), g\left(y_{n}\right)\right)\right\}$.

Lemma 4 Let $\left(F_{1}, F_{2}\right) \subseteq \llbracket n_{1} \rrbracket \times \llbracket n_{2} \rrbracket$, and let $T$ and $T^{\prime}$ be any two states of $\widehat{\mathrm{M}}_{\mathrm{F}_{1}, \mathrm{~F}_{2}}$ such that $T \rightarrow T^{\prime}$. Then, for any $a \in \llbracket n_{1} \rrbracket^{\llbracket n_{1} \rrbracket} \times \llbracket n_{2} \rrbracket^{\llbracket n_{2} \rrbracket}, \delta^{a}(T) \rightarrow \delta^{a}\left(T^{\prime}\right)$ or $\delta^{a}(T)=\delta^{a}\left(T^{\prime}\right)$.

Proposition 2 Let $\left(F_{1}, F_{2}\right) \subseteq \llbracket n_{1} \rrbracket \times \llbracket n_{2} \rrbracket$, and let $T, T^{\prime}$ be two states of $\mathrm{M}_{\mathrm{F}_{1}, \mathrm{~F}_{2}}$. If $T \stackrel{*}{\leftrightarrow} T^{\prime}$, then $T$ and $T^{\prime}$ are not distinguishable.

Proof: From Lemma 4 it is easy to see by a simple induction that, for any word $w$ if $T \rightarrow T^{\prime}$ then $\delta^{w}(T) \rightarrow \delta^{w}\left(T^{\prime}\right)$ or $\delta^{w}(T)=\delta^{w}\left(T^{\prime}\right)$. From Lemma 3, if $T \rightarrow T^{\prime}$, then $T \sim_{N e r} T^{\prime}$ in the sense of the Nerode equivalence. Thus, as $\stackrel{*}{\leftrightarrow}$ is the symmetric and transitive closure of $\rightarrow, T \stackrel{*}{\leftrightarrow} T^{\prime}$ implies $T \sim_{N e r} T^{\prime}$.

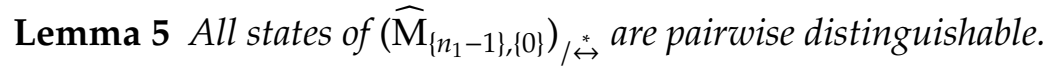

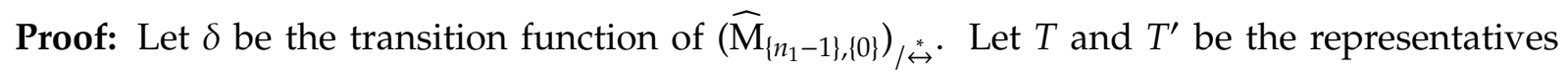
of two states of $\left(\widehat{\mathrm{M}}_{\left\{n_{1}-1\right\},\{0\}}\right)_{/ \stackrel{*}{\leftrightarrow}}$ such that $T \neq T^{\prime}$. Let $(i, j)$ be such that $T_{i, j} \neq T_{i, j}^{\prime}$. Suppose, for example that $T_{i, j}=1$. Take $\left\{i_{1}, \ldots, i_{\ell}\right\}=\left\{\alpha \mid T_{\alpha, j}^{\prime}=1\right\}$ and $\left\{j_{1}, \ldots, j_{p}\right\}=\{j\} \cup\left\{\beta \mid T_{i_{1}, \beta}^{\prime}=1\right\}$. We can see that :

1. By Lemma 2, lines $i_{1}, \ldots, i_{\ell}$ are the same, as they all have a cross on the column $j$. Columns $\left\{j_{1}, \ldots, j_{p}\right\}$ are also the same, as they all have a cross on line $i_{1}$. It follows that, if $\left(i^{\prime}, j^{\prime}\right) \in$ $\left(\left\{i_{1}, \ldots, i_{\ell}\right\} \times\left(\left\{0, \ldots, n_{2}-1\right\} \backslash\left\{j_{1}, \ldots, j_{p}\right\}\right)\right) \cup\left(\left(\left\{0, \ldots, n_{1}-1\right\} \backslash\left\{i_{1}, \ldots, i_{\ell}\right\}\right) \times\left\{j_{1}, \ldots, j_{p}\right\}\right)$, then $T_{i^{\prime}, j^{\prime}}^{\prime}=$ 0

2. $j \in\left\{j_{1}, \ldots, j_{p}\right\}$ and $i \notin\left\{i_{1}, \ldots, i_{\ell}\right\}$.

Let $f\left(i^{\prime}\right)=\left\{\begin{array}{ll}n_{1}-1 & \text { if } i^{\prime} \in\left\{i_{1}, \ldots, i_{\ell}\right\}, \\ 0 & \text { otherwise, }\end{array}\right.$ and $g\left(j^{\prime}\right)= \begin{cases}0 & \text { if } j^{\prime} \in\left\{j_{1}, \ldots, j_{p}\right\} \\ n_{2}-1 & \text { otherwise. }\end{cases}$

If $\left(f\left(i^{\prime}\right), g\left(j^{\prime}\right)\right)$ is in the final zone of $\mathrm{M}_{\left\{n_{1}-1\right\},\{0\}}$, then

$$
\left(i^{\prime}, j^{\prime}\right) \in\left(\left\{i_{1}, \ldots, i_{\ell}\right\} \times\left(\left\{0, \ldots, n_{2}-1\right\} \backslash\left\{j_{1}, \ldots, j_{p}\right\}\right)\right) \cup\left(\left(\left\{0, \ldots, n_{1}-1\right\} \backslash\left\{i_{1}, \ldots, i_{\ell}\right\}\right) \times\left\{j_{1}, \ldots, j_{p}\right\}\right),
$$

and so the first point above gives us $T_{i^{\prime}, j^{\prime}}^{\prime}=0$. 

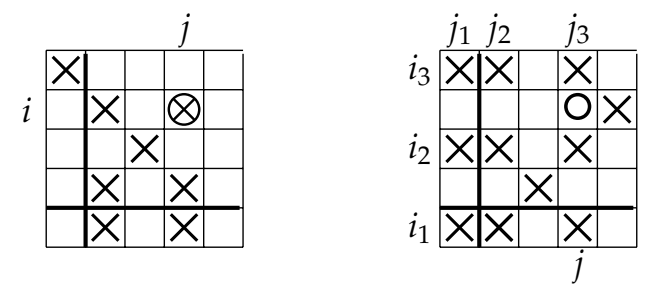

Figure 7: An example of two tableaux $T$ and $T^{\prime}$

Therefore, $\delta^{(f, g)}\left(T^{\prime}\right)$ has only at most two crosses, one in $\left(n_{1}-1,0\right)$ and one in $\left(0, n_{2}-1\right)$, and it is not final. However, the second point above and the fact that $T_{i, j}=1$ gives us that $\delta^{(f, g)}(T)_{0,0}=1$, which means that $\delta^{(f, g)}(T)$ is final. Thus, $T$ and $T^{\prime}$ are distinguishable.

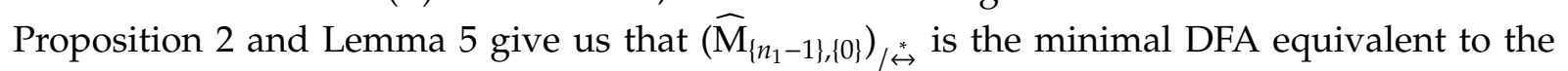
DFA $\widehat{\mathrm{M}}_{\left\{n_{1}-1\right\},\{0\}}$. The following corollary stems from this assertion combined with Lemma 1 .

Corollary $1\left(\widehat{\mathrm{M}}_{\left\{n_{1}-1\right\},\{0\}}\right)_{/ \leftrightarrow}^{*}$ is the minimal DFA equivalent to $\mathrm{M}_{\left\{n_{1}-1\right\},\{0\}}$.

\subsection{Computing the state complexity of the language recognized by $\mathrm{M}_{\left\{n_{1}-1\right\},\{0\}}$}

The number of right-triangle free tableaux $T$ of size $\llbracket n_{1} \rrbracket \times \llbracket n_{2} \rrbracket$ such that, if $T$ has a cross in the final zone of $\mathrm{M}_{\left\{n_{1}-1\right\},\{0\}}$, then $T$ has cross at $(0,0)$ is exactly $2 \alpha_{n_{1}-1, n_{2}-1}+\alpha_{n_{1}, n_{2}}^{\prime}$ where $\alpha_{x, y}$ is the number of right-triangle free tableaux of size $x \times y$ and $\alpha_{x, y}^{\prime}$ the number of right-triangle free tableaux of size $x \times y$ having a cross in $(0,0)$. Therefore,

Lemma 6 The state complexity of $\mathrm{L}\left(\mathrm{M}_{\left\{n_{1}-1\right\},\{0\}}\right)$ is $2 \alpha_{n_{1}-1, n_{2}-1}+\alpha_{n_{1}, n_{2}}^{\prime}$.

Closed formulas for $\alpha(x, y)$ and $\alpha^{\prime}(x, y)$ are given in Corollary 20 and Proposition 22 of [4].

In the next subsection, we prove that $\left(\left\{n_{1}-1\right\},\{0\}\right)$ is a couple of final states that maximizes the size of the minimal DFA associated to any $\mathrm{M}_{F_{1}, F_{2}}$, with $\left(F_{1}, F_{2}\right) \subseteq \llbracket n_{1} \rrbracket \times \llbracket n_{2} \rrbracket$.

\subsection{Maximizing the state complexity of $\Theta$ applied to monster 2-languages}

Let $\mathcal{T}$ be the set of right-triangle free tableaux of size $n_{1} \times n_{2}$. For all $\left(F_{1}, F_{2}\right) \subseteq \llbracket n_{1} \rrbracket \times \llbracket n_{2} \rrbracket$, let

$$
\mathcal{T}_{F_{1}, F_{2}}=\#\left(\widehat{\mathrm{M}}_{F_{1}, F_{2}}\right)_{/ \stackrel{\leftrightarrow}{\leftrightarrow}}=\#\left\{T \in \mathcal{T} \mid T \text { has a cross in the final zone implies } T_{0,0}=1\right\} .
$$

We show that :

Lemma 7 For any $F_{1} \times F_{2} \subseteq \llbracket n_{1} \rrbracket \times \llbracket n_{2} \rrbracket$ such that $F_{1}, F_{2} \neq \emptyset$ and $F_{1} \neq \llbracket n_{1} \rrbracket, F_{2} \neq \llbracket n_{2} \rrbracket, \mathcal{T}_{F_{1}, F_{2}} \leq$ $\mathcal{T}_{\left\{n_{1}-1\right\},\{0\}}$.

Therefore, by Remark 2, Proposition 2, and Corollary 1, for any $F_{1} \times F_{2} \subseteq \llbracket n_{1} \rrbracket \times \llbracket n_{2} \rrbracket$ such that $F_{1}, F_{2} \neq \emptyset$ and $F_{1} \neq \llbracket n_{1} \rrbracket, F_{2} \neq \llbracket n_{2} \rrbracket$,

$$
\#_{\min }\left(\mathrm{M}_{F_{1}, F_{2}}\right) \leq \#\left(\left(\widehat{\mathrm{M}}_{F_{1}, F_{2}}\right)_{/ \leftrightarrow}\right)=\mathcal{T}_{F_{1}, F_{2}} \leq \mathcal{T}_{\left\{n_{1}-1\right\},\{0\}}=\#\left(\left(\widehat{\mathrm{M}}_{\left\{n_{1}-1\right\},\{0\}}\right)_{\mid \leftrightarrow}\right)=\#_{\min }\left(\mathrm{M}_{\left\{n_{1}-1\right\},\{0\}}\right) .
$$

The cases where $F_{1}=\emptyset$ or $F_{2}=\emptyset$ or $F_{1}=\llbracket n_{1} \rrbracket$ or $F_{2}=\llbracket n_{2} \rrbracket$ are easy and proven by :

Lemma 8 If $F_{1}=\emptyset$ or $F_{2}=\emptyset$ or $F_{1}=\llbracket n_{1} \rrbracket$ or $F_{2}=\llbracket n_{2} \rrbracket$, then $\#_{\min }\left(\mathrm{M}_{F_{1}, F_{2}}\right) \leq \#_{\min }\left(\mathrm{M}_{\left\{n_{1}-1\right\},\{0\}}\right)$.

Therefore, by Theorem 3 and Lemma 6 ,

Theorem 5 The state complexity of $\circledast$ is $2 \alpha_{n_{1}-1, n_{2}-1}+\alpha_{n_{1}, n_{2}}^{\prime}$ i.e. for all $n_{1}, n_{2} \in \mathbb{N}^{*}, s c \circledast\left(n_{1}, n_{2}\right)=$ $2 \alpha_{n_{1}-1, n_{2}-1}+\alpha_{n_{1}, n_{2}}^{\prime}$. 


\section{Witnesses with a bounded alphabet size}

We now prove that there is a finite-bounded-alphabet witness. Let $n_{1}, n_{2}$ be two positive integers and let $\left(\mathbb{M}_{1}, \mathbb{M}_{2}\right)=\operatorname{Mon}_{n_{1}, n_{2}}^{\left\{n_{1}-1,\{0\}\right.}$. Recall that the letters of $\operatorname{Mon}_{n_{1}, n_{2}}^{\left\{n_{1}-1\right\},\{0\}}$ are couples of mappings and that $\mathbb{1}$ is the identities bot in $\llbracket n_{1} \rrbracket$ and in $\llbracket n_{2} \rrbracket$. Let $B_{1}$ and $B_{2}$ be the DFAs obtained by restricting the letters of respectively $\mathbb{M}_{1}$ and $\mathbb{M}_{2}$ to the alphabet

$$
\begin{aligned}
\Sigma^{\prime}= & \left\{\left(\left(0, \ldots, n_{1}-2\right), \mathbb{1}\right),\left(\left(1, \ldots, n_{1}-2\right), \mathbb{1}\right),\left(\mathbb{1},\left(1, \ldots, n_{2}-2\right)\right),\left(\left(1, \ldots, n_{1}-1\right), \mathbb{1}\right),\right. \\
& \left(\mathbb{1},\left(1, \ldots, n_{2}-1\right)\right),\left(\left(0, n_{1}-1\right), \mathbb{1}\right),\left(\mathbb{1},\left(0, n_{2}-1\right)\right),((0,1),(0,1)),((0,1), \mathbb{1}),(\mathbb{1},(0,1)), \\
& \left.\left(\left(n_{1}-2, n_{1}-1\right), \mathbb{1}\right),\left(\left(\begin{array}{l}
1 \\
0
\end{array}\right), \mathbb{1}\right),\left(\mathbb{1},\left(\begin{array}{l}
1 \\
0
\end{array}\right)\right),\left(\left(\begin{array}{l}
n_{1}-2 \\
n_{1}-1
\end{array}\right), \mathbb{1}\right),\left(\mathbb{1},\left(\begin{array}{l}
n_{2}-2 \\
n_{2}-1
\end{array}\right)\right),\left(\left(\begin{array}{c}
n_{1}-1 \\
0
\end{array}\right), \mathbb{1}\right),\left(\mathbb{1},\left(\begin{array}{c}
n_{2}-1 \\
0
\end{array}\right)\right)\right\} .
\end{aligned}
$$

Let $B=\operatorname{St} \mathfrak{X}\left(B_{1}, B_{2}\right)$, and $\widehat{B}$ be the DFA obtained by restricting $B$ to states $T$ such that, if $T$ has a cross in the final zone of $\mathrm{M}_{\left\{n_{1}-1\right\},\{0\}}$, then $T$ has cross at $(0,0)$. The DFA $A=\widehat{B}_{/ \leftrightarrow}$ is obtained by restricting the letters of $\widehat{\mathrm{M}}_{\left\{n_{1}-1\right\},\{0\}}$ to the alphabet $\Sigma^{\prime}$. We are going to show that $A$ is minimal.

Let us recall that all letters of $\Sigma^{\prime}$ can be seen as a function acting on tableaux. Every word $w$ of $\Sigma^{\prime}$ acts on a tableau $T$ by applying the composition of all letters of $w$ to $T:$ if $w=a_{1} \ldots a_{n}$, define $w(T)=a_{n} \circ \ldots \circ a_{1}(T)$. When it exists, we denote by $w^{-1}$ the inverse function of $a_{n} \circ \ldots \circ a_{1}$. Let $\delta$ be the transition function of $B$. We first notice that $w(T)$ is not necessarily equivalent to $T^{\prime}=\delta^{w}(T)$ since $(0,0)$ is in $T^{\prime}$ if $T^{\prime}$ has a cross in the final zone. We denote by $w[i, j]$ the subword $a_{i} \cdots a_{j}$. By convention, if $\mathrm{j}<\mathrm{i}, w[i, j]=\varepsilon$. The proof of the following lemma is easy by induction. Lemma 9 Let $w$ be a word of $\Sigma^{\prime}$, and $T$ be a state of $B$. If, for any integer $k<|w|$, we have $(w[1, k](T))_{0,0}=1$ or $w[1, k](T)$ has no cross in the final zone, then $\delta^{w}(T)=w(T)$.

Lemma 10 All the states of $\widehat{B}$ are accessible.

Proof: As the induction is the same as in Lemma 1, we only focus on cases of this previous lemma where the letters used are not in $\Sigma^{\prime}$.

- If $T^{\prime}$ has no cross in the final zone, according the previous remark, we have only to examine the case where $T_{n_{1}-1,0}^{\prime}=0$. Let $(i, j)$ be the index of a cross of $T^{\prime}$. Let $w=\left((\mathbb{1},(0,1))\left(\left(0, \ldots, n_{1}-\right.\right.\right.$ $2), \mathbb{1})^{i}\left(\mathbb{1},\left(1, \ldots, n_{2}-1\right)\right)^{j-1}$ and let $T=w^{-1}\left(T^{\prime}\right)$. We have $T_{0,0}=1$ and for all $1<k<|w|$, for all $(i, j) \neq\left(n_{1}-1,0\right)$ such that $i=n_{1}-1$ and $j=0,(w[1, k](T))_{i, j}=\left(w[k,|w|-1]^{-1}\left(T^{\prime}\right)\right)_{i, j}=0$. Thus, by Lemma $9, \delta^{w}(T)=T^{\prime}$. We also have $T<T^{\prime}$ since $\# T=\# T^{\prime}$ and $\#_{\mathrm{nf}} T<\#_{\mathrm{nf}} T^{\prime}$.

- $T^{\prime}$ has a cross in the final zone other than $(0,0)$. Let $(i, j)$ be such a cross. We distinguish two cases.

- If $j=0$, we consider the word $w_{1}=\left(\left(1, \ldots, n_{1}-2\right), \mathbb{1}\right)^{i}$. Let $T^{\prime \prime}$ be the tableau obtained from $w_{1}^{-1}\left(T^{\prime}\right)$ by deleting the cross at $(0,0)$ and let $w_{2}=((0,1), \mathbb{1})$ and $T=w_{2}\left(T^{\prime \prime}\right)$. It is easy to see that $T^{\prime}=w_{1}\left(\delta^{w_{2}}(T)\right)$. By Lemma 9 , we have $T^{\prime}=w_{1}\left(\delta^{w_{2}}(T)\right)=\delta^{w_{2} w_{1}}(T)$ in $\widehat{\mathrm{M}}_{\left\{n_{1}-1\right\},\{0\}}$ and $T<T^{\prime}$ as $\# T<\# T^{\prime}$.

- Otherwise define $w_{1}=\left(\left(1, \ldots, n_{1}-1\right), \mathbb{1}\right)^{i-1}\left(\mathbb{1},\left(1, \ldots, n_{2}-1\right)\right)^{j-1}$. Let $T^{\prime \prime}$ be tableau obtained from $w_{1}^{-1}\left(T^{\prime}\right)$ by deleting the cross at $(0,0)$. It means that $T^{\prime}=w_{1}\left(T^{\prime \prime}\right) \cup\{(0,0)\}$. Let $w_{2}=((0,1),(0,1))$ and $T=w_{2}\left(T^{\prime \prime}\right)$. Then we have $\delta^{w_{2}}(T)=T^{\prime \prime} \cup\{(0,0)\}$ or $\delta^{w_{2}}(T)=$ $T^{\prime \prime}$.

* If $\delta^{w_{2}}(T)=T^{\prime \prime} \cup\{(0,0)\}$, then by Lemma 9 , as $w_{1}$ does not change the first line and the first column we have $\delta^{w_{2} w_{1}}(T)=\delta^{w_{1}}\left(\delta^{w_{2}}(T)\right)=w_{1}\left(\delta^{w_{2}}(T)\right)=w_{1}\left(\left(T^{\prime \prime} \cup\{0,0)\right\}\right)=$ $w_{1}\left(T^{\prime \prime}\right) \cup\{(0,0)\}=T^{\prime}$. 
* If $\delta^{w_{2}}(T)=T^{\prime \prime}$, then we set $k=\min \left\{l<|w| \mid \delta^{\left(w_{1}[1, l]\right.}\left(T^{\prime \prime}\right)_{0,0}=1\right\}$. For all integer $l<k$, the tableau $\delta^{w_{1}[1, l]}\left(T^{\prime \prime}\right)$ has no cross in the final zone, and we can apply Lemma 9 . and $w_{2}\left(w_{1}[1, l](T)\right)=\delta^{w_{2} w_{1}}[1, l](T)$. Furthermore $\delta^{w_{2} w_{1}[1, k]}(T)=\left(w_{1}[1, k]\right)\left(\delta^{w_{2}}(T)\right) \cup$ $\{(0,0)\}$ and as letters of $w_{1}\left[k+1,\left|w_{1}\right|\right]$ do not change the first line and the first column, we have $\delta^{w_{2} w_{1}}(T)=w_{1}\left[k+1,\left|w_{1}\right|\right]\left(w_{1}[1, k]\left(\delta^{w_{2}}(T)\right) \cup\{(0,0)\}\right)=w_{1}\left(\delta^{w_{2}}(T)\right) \cup$

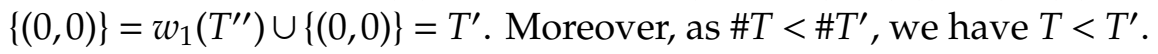

- The only cross of $T^{\prime}$ which is in the final zone is $(0,0)$. According to the first sentence of the proof, we have only to consider the case where there does not exist $j$ such that $T_{0, j}^{\prime}=1$ and $T_{n_{1}-1,0}^{\prime}=0$. It follows that there exists $(i, j) \neq\left(n_{1}-1,0\right)$ such that $i \neq 0$ and $T_{i, j}^{\prime}=1$. Let $w=\left(\left(1, \ldots, n_{1}-1\right), \mathbb{1}\right)^{i}$ and let $T=w^{-1}\left(T^{\prime}\right)$. By Lemma 9 , as for each proper prefix $w^{\prime}$ of $w$, $\left(w^{\prime}(T)\right)_{0,0}=1$, we have $\delta^{w}(T)=T^{\prime}$ in $B$, and $T<T^{\prime}$ as $\#_{\mathrm{nf}} T<\#_{\mathrm{nf}} T^{\prime}$.

Similarly, the following lemma is obtained by simulating with letters in $\Sigma^{\prime}$ the transition functions used in Lemma5,

Lemma 11 All states of A are pairwise distinguishable.

Lemma 10 and 11 imply that $A$ is minimal and that the following theorem holds.

Theorem 6 The couple $\left(B_{1}, B_{2}\right)$ is a witness for the operation $\circledast$.

\section{Conclusion}

We have given the state complexity of the star of symmetrical difference and have provided a witness with a constant alphabet size. We know that the bounded size of the alphabet that we exhibit is not optimal, but it simplifies the proof given. Moreover, proving the optimality of a bound seems out of reach for now and would necessitate to introduce new tools.

One of our future works will be to generalize the method used here to a whole well-defined class of operations, in order to provide a witness with bounded alphabet size for all of them. Acknowlegedments This work is partially supported by the projects MOUSTIC ( ERDF/GRR) and ARTIQ (ERDF/RIN)

\section{References}

[1] Janusz A. Brzozowski (2013): In Search of Most Complex Regular Languages. Intern. J. of Foundations of Comp. Sc. 24(6), pp. 691-708. Available at http://dx.doi.org/10.1142/S0129054113400133

[2] Janusz A. Brzozowski, Galina Jirásková, Bo Liu, Aayush Rajasekaran \& Marek Szykula (2016): On the State Complexity of the Shuffle of Regular Languages. In: Descriptional Complexity of Formal Systems - 18th IFIP WG 1.2 International Conference, DCFS 2016, Bucharest, Romania, July 5-8, 2016. Proceedings, pp. 73-86, doi 10.1007/978-3-319-41114-9_6.

[3] Pascal Caron, Edwin Hamel-De le court, Jean-Gabriel Luque \& Bruno Patrou (2018): New tools for state complexity. CoRR abs/1807.00663. Available at http://arxiv.org/abs/1807.00663.

[4] Pascal Caron, Jean-Gabriel Luque, Ludovic Mignot \& Bruno Patrou (2016): State Complexity of Catenation Combined with a Boolean Operation: A Unified Approach. Int. J. Found. Comput. Sci. 27(6), pp. 675-704, doi $10.1142 / S 0129054116500234$.

[5] Pascal Caron, Jean-Gabriel Luque \& Bruno Patrou (2016): State complexity of multiple catenation. CoRR abs/1607.04031. Available at http://arxiv.org/abs/1607.04031. 
[6] Pascal Caron, Jean-Gabriel Luque \& Bruno Patrou (2017): State complexity of catenation combined with boolean operations. CoRR abs/1707.03174. Available at http://arxiv.org/abs/1707.03174.

[7] Bo Cui, Yuan Gao, Lila Kari \& Sheng Yu (2011): State Complexity of Two Combined Operations: Catenation-Union and Catenation-Intersection. Int. J. Found. Comput. Sci. 22(8), pp. 1797-1812. Available at http://dx.doi.org/10.1142/S0129054111009045.

[8] Sylvie Davies (2018): A General Approach to State Complexity of Operations: Formalization and Limitations. Developments in Language Theory, doi:10.1007/978-3-319-98654-8_21.

[9] Michael Domaratzki (2002): State Complexity of Proportional Removals. Journal of Automata, Languages and Combinatorics 7(4), pp. 455-468, doi 10.25596/jalc-2002-455.

[10] Michael Domaratzki \& Alexander Okhotin (2009): State complexity of power. Theoretical Computer Science 410(24), pp. 2377 - 2392, doi $10.1016 / j$.tcs.2009.02.025. Available at http://www. sciencedirect.com/science/article/pii/S0304397509001820 Formal Languages and Applications: A Collection of Papers in Honor of Sheng Yu.

[11] Yuan Gao, Nelma Moreira, Rogério Reis \& Sheng Yu (2017): A Survey on Operational State Complexity. Journal of Automata, Languages and Combinatorics 21(4), pp. 251-310, doi 10.25596/jalc-2016-251.

[12] Yuan Gao, Kai Salomaa \& Sheng Yu (2008): The State Complexity of Two Combined Operations: Star of Catenation and Star of Reversal. Fundam. Inf. 83(1-2), pp. 75-89. Available at http://dl.acm.org/ citation.cfm?id=1377804.1377812,

[13] J. E. Hopcroft \& J. D. Ullman (1979): Introduction to Automata Theory, Languages and Computation. Addison-Wesley, Reading, MA.

[14] Jozef Jirásek, Galina Jirásková \& Alexander Szabari (2005): State complexity of concatenation and complementation. Int. J. Found. Comput. Sci. 16(3), pp. 511-529. Available at http://dx.doi.org/ 10.1142/S0129054105003133.

[15] Galina Jirásková (2005): State complexity of some operations on binary regular languages. Theor. Comput. Sci.330(2), pp. 287-298. Available at http://dx.doi.org/10.1016/j.tcs.2004.04.011

[16] Galina Jirásková \& Alexander Okhotin (2008): State complexity of cyclic shift. ITA 42(2), pp. 335-360, doi 10.1051/ita:2007038.

[17] Galina Jirásková \& Alexander Okhotin (2011): On the State Complexity of Star of Union and Star of Intersection. Fundam. Inform. 109(2), pp. 161-178. Available at http://dx.doi.org/10.3233/ FI-2011-502.

[18] Arto Salomaa, Kai Salomaa \& Sheng Yu (2007): State complexity of combined operations. Theor. Comput.Sci.383(2-3), pp.140-152. Available athttp://dx.doi.org/10.1016/j.tcs.2007.04.015.

[19] Sheng Yu (2001): State Complexity of Regular Languages. Journal of Automata, Languages and Combinatorics 6(2), p. 221, doi:10.25596/jalc-2001-221. 